\title{
CONSTRUCTION OF THE INTEGRATED COMPUTER-AIDED DESIGN PLATFORM OF HYDRAULIC PRESSURE SUPPORT
}

\author{
Tan Heng ${ }^{1,2, a}$, Li Chunyu ${ }^{1, b}$, Tan Tongde ${ }^{1, c}$ \\ ${ }^{1}$ School Of Information Engineering, Zhengzhou University, Zhengzhou 450001, China; \\ ${ }^{2}$ Henan Provincial Key Lab on Information Networking, Zhengzhou 450052, China;

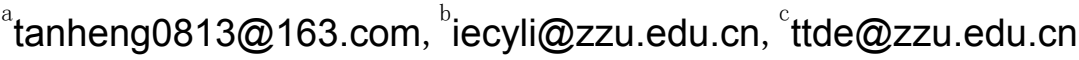

Keywords: Hydraulic pressure support design, Integration, Networking, Visual, Computer-aided design.

\begin{abstract}
As a key device to coal mining, the hydraulic pressure support is important for the safety of coal mine. Currently, most of design software for hydraulic pressure support are stand-alone version and only capable of fulfilling partial design work. They have been used for a long time and lacked software maintenance, moreover, which use different development languages and database, structure of data table. Current using software become networking and integration for purpose, and also for the requirement of Zhengzhou coal mine machinery group co.,ltd, utilizing cloud technology, specific 10 design software is SaaS and networking, which are in order to build the overall design platform of hydraulic pressure support. According to the requirement of company designing accessories, a visual design platform for accessories of hydraulic pressure support based on $\mathrm{B} / \mathrm{S}$ structure is presented, plus collaborates with overall design platform as one-the integrated computer-aided design platform of hydraulic pressure support. Finally, the feasibility and validity of the presented method is demonstrated.
\end{abstract}

\section{Introduction}

There are two steps of designing hydraulic support in China, which are overall design and detail design. In period of overall design, firstly, it determines working resistance of hydraulic support that depending on geological condition and working condition of hydraulic support. Then, basing on working resistance calculates size of main component in hydraulic support. In period of detail design, on the basis of sizes and design experiences and inventory of main components determine the sizes and shapes of other parts; finally, through all mentioned measure finishes up the whole machine design.

Currently, hydraulic support design uses many kinds of CAD (computer aided design) software to design part in China. Those software have no connectivity between each other, and most of them are for stand-alone usage, engineer can't share outcomes of design. How to integrate and network current using software, for strengthening team work, is a question need to be solved.

\section{Research background}

Back in 1990s, information technology made a figure in machinery manufacturing. To conclude outcome of that information technology apply in advanced manufacturing from different perspectives, and combine with current situation of coal mine machinery informatization, to be innovative, open-minded, be constructive to fuse advanced manufacturing informatization into stages such as, optimal design, manufacturing, and management, which have a significant meaning to coal mine machinery[1].

With information technology extensively applying in coal mine machinery design, it brings lots of convenience along with inconvenience and so. In period of overall, engineers mainly analyze performance(kinematics, statics, bearing capacity),structure(strength analysis of key supporting parts).In period of overall, engineers need to calculate and analyze in multiple aspects, enterprises 
use design software exclusive for one aspect so far. Due to high professional and specificity, there is not software for sale that can finish all works. At present, machinery enterprises collaborate with universities on developing software being competent for specific analysis, those software can only finish own assignment and can't cooperate, and most of them haven't good after-sale service, no to mention update and patch. However, to develop a new software from scratch that, no doubt, will bring a financial burden. Therefore, How to network current-using software, let them cooperate in period of overall design, which becomes an urgent requirement.

The emergence of cloud computing technology provides a new service platform with integrated, open, virtual and autonomous characteristics[2] which can effectively solve the problem of resource sharing and collaboration in the field of product diversification in the current manufacturing field. Based on cloud computing technology, the design and manufacturing and application system's support space spread from a single enterprise to the group, and then to the whole society[3], leading to the formation of private cloud or public cloud, in the product platform development process of product design, simulation, testing and other aspects of the application need a large number of data and software resources, so the reasonable allocation and full use of the massive data and software resources is a core issue of the product design cloud service[4].

Private cloud refers to the enterprise or organization to build and use the cloud computing environment, which is often not open to the outside world, is exclusive, closed a private cloud computing environment. Only enterprise or organization member has access to the private cloud internal resources and related data, external users (enterprises and individuals) have no permission so that can't hire a private cloud resources or access to private cloud data[5].

In this paper, through the establishment of enterprise internal private cloud platform, using unified interface to networking, which integrates the existing software, using a variety of techniques to achieve the communication between heterogeneous software, then builds up the high compatibility of the hydraulic support design platform.

In the past, in design of hydraulic support, the design of parts has not been perfect, and the general use of the Pro/E software for the force analysis of small parts on the support is the very limited. In this paper, we study visualize and virtualize methods of the design of the parts. By combining the Microsoft Silverlight development tool, the 3D-simulation results are presented effectively and efficiently. As for the design data is stored in the internal private cloud for easy sharing and management. This method does not need actual experiment model, which saves the resources and improves the design precision and strengthen the competitiveness of the enterprises.

\section{Platform framework}

Currently design software are used by enterprises, which use different languages, different databases, different support libraries, mostly heterogeneous software. And some of the software has been a network version, as there are their own authentication mechanism that is impossible to change the existing software certification. At the same time, users also want to have the software to collaborate.

Given this situation, enterprises usually only get three choices: One is to abandon the original local application, adopt a holistic solution (but it will lead to duplication of investment, also due to the operation habit change and data preparation work, will make the users of the original system in the implementation process to produce psychological conflict); second while retaining the original local application, to introduce new applications, not integration, integration of partial data and process using at outside of system(but it will lead to the emergence of information isolated island, increased human error and increase the input workload); three is to retain the original local application, and introduce a new system, followed by the one or two software companies cooperation to achieve integration[6]. We use the enterprise private cloud platform, integrate the existing software in the cloud platform, which remove the differences of these heterogeneous software, users just consider the own design work when use it, the platform as a kind of SaaS(Software as a Service). Place different software on the platform, and install various support libraries and software. In the platform, the data exchange layer is set up, and the data exchange 
layer is stored and exchanged data. The user need not care about the structure of the lower layer. For the reuse of existing software, develop a function module call the interface for communication between the client end and software, the user through the module to use of the platform's open application.

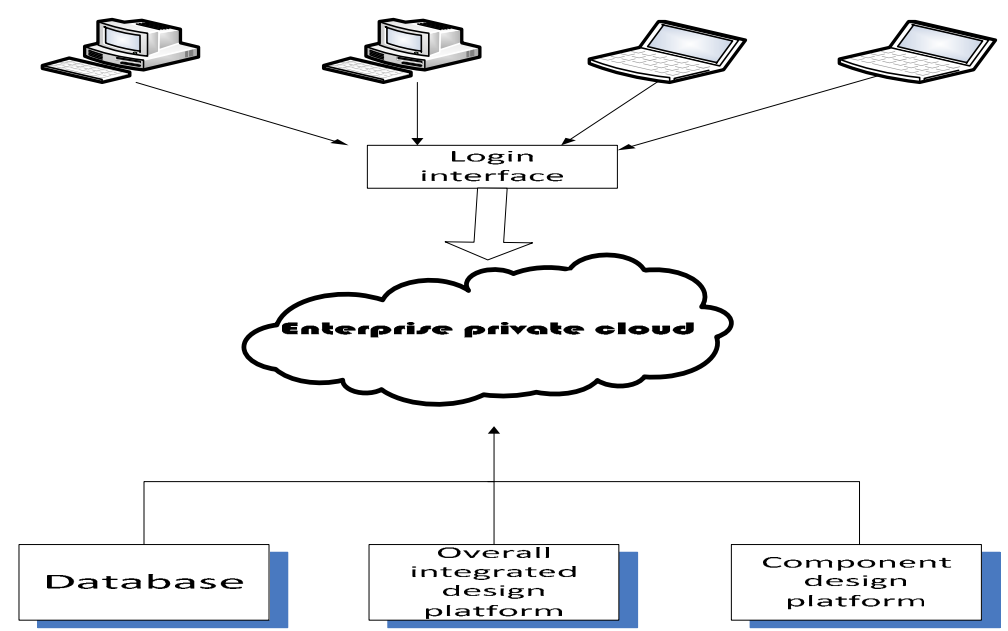

Fig. 1 the structure of connection

Cloud platform uses a domain control server. All the clients are added to the platform, so that each client end works in the same network routing environment.

In order to make full use of the function of the existing software and the newly developed software, a unified access is built on the client end. The client end uses a unified access interface, a collection of existing software and the newly developed software interface. The interface can choose to use the overall design platform or component design platform.

\section{Function of the integrated design platform of hydraulic pressure support}

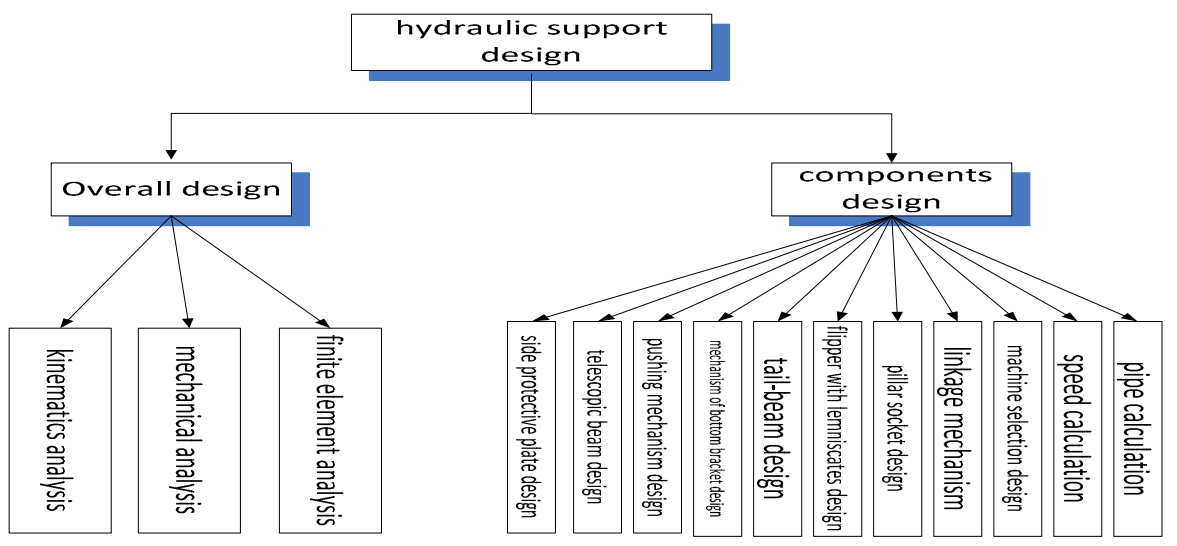

Fig. 2 function picture 


\section{Design platform core process}

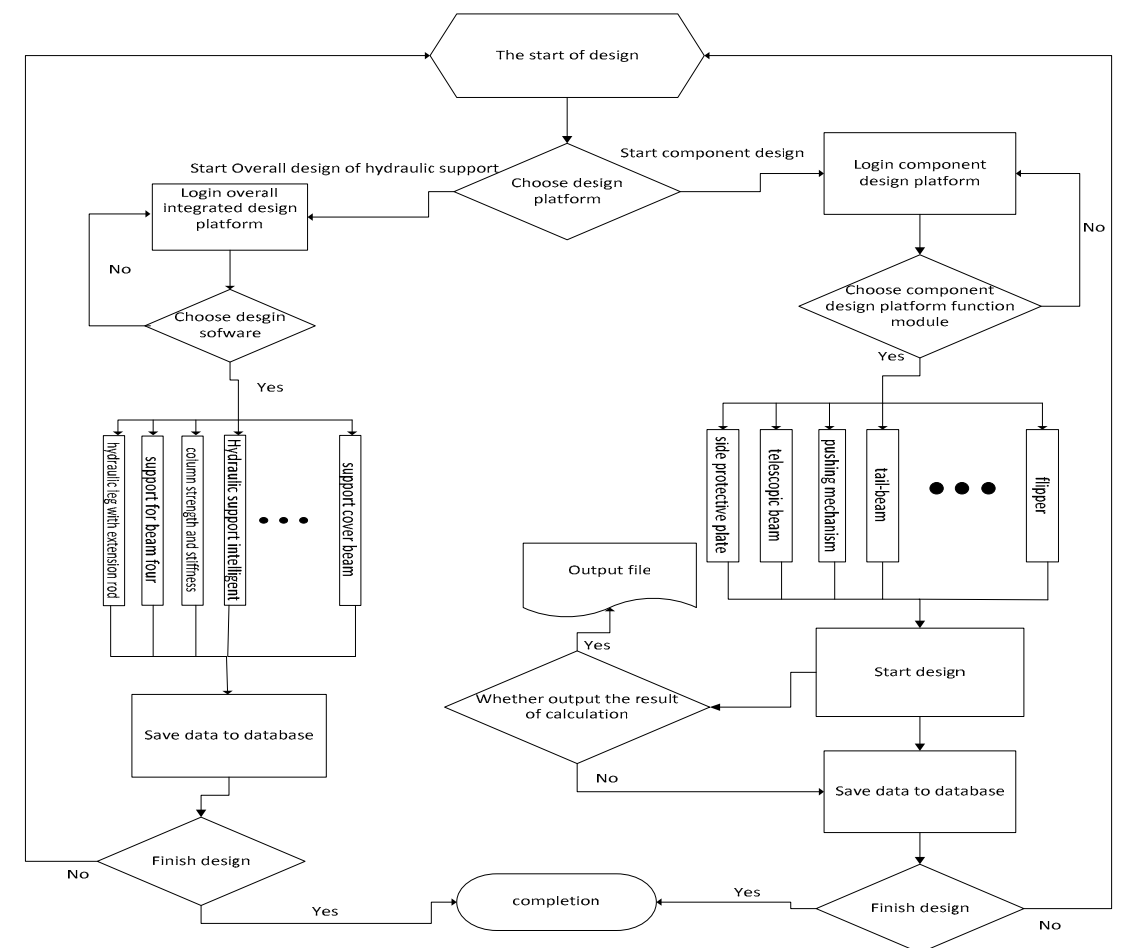

Fig.3 Overall design flow chart

Before the design work, first, we must know it will be the overall design or components design, then to choose what design platform. After platform selection, we can carry out the relevant design work. Through the combining use of the two platforms, data store, greatly facilitates the design staff's design.

\section{The construction of overall integrated design platform}

\subsection{Overall integrated design platform}

Hydraulic support intelligent computer aided design system, design system of hydraulic leg with extension rod, support for beam four bar design version 1.0, hydraulic column strength and stiffness analysis, hydraulic support cover beam analysis system and the coal wall stability analysis, low caving coal hydraulic support comprehensive analysis software, analysis of the relationship between support and surrounding rock, the stability of hydraulic support, hydraulic support optimization design.

Design system of hydraulic leg with extension rod calculates the structural parameters of the leg and the column strength. Support for beam four bar design calculates motion simulation, stress analysis and travel length of the four bar of support for beam. Hydraulic column strength and stiffness analysis applies to the analysis of strength and rigidity of double telescopic column. Other software have similar specific usage, will not be described in detail.

Those 10 software is mainly used to design the overall framework of hydraulic support, applying kinematics analysis, mechanical analysis, finite element analysis, which is the basis for the design of hydraulic support.

\subsection{Platform technical difficulties}

Cloud platform application layer is SaaS mode, that is, the oriented-to-users services. SaaS service mode, simple and flexible, once deployed, multiple entrances. As long as connecting in the work network, users can use the service anytime and anywhere. In order to use the cloud software as SaaS mode, need to test 10 small software hardware and software environment. Because of the different kinds of software functions, the different use of methods, the different software prerequisite environment, so the need to build the software environment for the client and the cloud at first. After the analysis of the 10 software, their Framework and prerequisite are found out, such 
as: matrix VB, some use the $\mathrm{OCX}($ Object Linking and Embedding Control eXtension), if program have lost OCX, need to find missing ones and then re-register in the system. At the same time, the client's operating system version is the different, such as 32-bit, 64-bit operating system, and most of systems are pirated edition or simplified and partly missing dynamic link files. For 64 bit system is not compatible with 32-bit program problem, the program is unified and placed in system compatible folder that solves 32-bit applications may not work on the new 64 bit system.

According to the mentioned problems of the 10 software, producing a support package install in the client and have the required framework, software package, dynamic documents, which are concentrated together.

The second difficulty is that the existing software's encryption mechanism. A number of software among 10 software, in order to prevent unauthorized use of multiple computers, carries out encryption. After the research of the encryption mechanism, the component is designed to automatically upload the encrypted file corresponding to the client from the local to the private cloud platform. Thus the effect of encryption on the integration and network is solved. In aspect of data, the existing software to use a variety of database, for example, dBASE, access, SQL Server. The data may be used by other programs or later used in the design phase of parts. In order to eliminate the structural differences between heterogeneous databases, according to the actual development situation to design a data API interface receiving data from existing software, it is then converted the received data to identify the database file, so as to avoid the two heterogeneous database direct communication leads to the problem of data. After data conversion, the file format can also be read and stored in the cloud database in later phase of designing part, which is quite convenient for data owner and others using. Cloud platform workloads can meet the $99 \%$ design work that designer simultaneously taking the cloud data operation.

\section{Design for components}

\subsection{Component design platform}

In the initial design stage of component, it can't use the Pro/E, CAD alike the general engineering design software for preliminary mechanics analysis. Therefore using Microsoft Silverlight technology develops a calculation platform for the initial design of components. Because of a large number of parameters at design of parts, each repeated input, will undoubtedly affect the design efficiency, so the platform by using SQL Server database data do data support for different parts such as: side protective plate of hydraulic support, hydraulic adjustment mechanism of bottom bracket. Through the unified data interface to read the list of parts stored in the cloud platform database, directly delivered to the design page input box. Silverlight is a fusion of Microsoft's multiple technologies Web rendering technology. It provides a development framework, and through the use of image layer technology based on vector, supports the seamless integration of any size image, based on ASP. Net and Ajax web development environment to achieve a seamless connection[7].

Using Silverlight powerful graphics processing ability can make the design of parts according to different parameters, which shows in shape of three-dimensional graphics in real-time on the browser design graphics interface. In the component design platform, the $3 \mathrm{D}$ virtual model is supported by the knowledge of graphic geometry, which supports 360 degrees of push, pull and shift. The software structure of component design platform is the use of three layer software architecture. Through Web Service technology and cloud server communicates. 


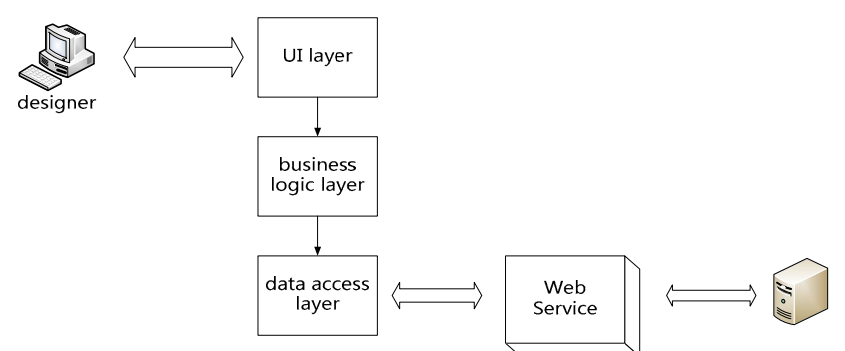

Fig. 4 Three layer architecture software design and Web Service

The three layer architecture is presented as presentation layer, business logic layer, data access layer.

The first layer is the UI (Interface User) layer, which is utilized by the designer. The entity layer in the lower layers creates a virtual representation class container through the parameters for designing the components used in the presentation layer. The business logic layer transfers designer's operation in presentation layer to data access layer being as the intermediate layer to the database for interactive operation, save or read data. The three layer structure method makes the design of the program more standardized, the structure is clearer, and the coupling degree between different layers is reduced. For the performance layer, the client end sends a request to the server, through the server's security authentication and authorization, you can get the services you need. For the interface layer, users can call server-end program interface in their own applications through Web service, RMI (remote method invocation, remote method call) et cetera, to get their required data and information[8].

In communication, the communication between clients and server use the Web Service technology. Web Service uses HTTP protocol communications used by most of browsers, being compatible with the vast majority of firewall. Web Service uses XML (Markup Language Extensible) to represent the basic data format, which is independent of the platform, and has nothing to do with the manufacturer, is the standard data type. Thus it can transmit between different platforms by the network through the SOAP protocol in the ASCII text mode. Using Web Service only transmits design parameters, and parameters will be serialized which makes the compression rate is greater than $70 \%$, greatly reducing the transmission pressure of the network.

\subsection{Component design platform function module.}

For configuration, designers login the component design platform deployed in the private cloud.

Through the login interface to query the user information in the cloud database, to provide different functions based on different users' permissions. There are 11 function modules in the platform.

1. Side protective plate design 2.telescopic beam design 3.pushing mechanism design 4. mechanism of bottom bracket design 5.tail-beam design 6.flipper with lemniscates design 7.pillar socket design 8.linkage mechanism 9.machine selection design 10.speed calculation 11.pipe calculation

The first 9 are components design, the last two is the calculation of the mechanics.

(1) Side protective plate design

The design of the side protective plate displays in two sections: the design of the side protective plate board and the calculation of the total force.

Using Silverlight technology to display the outline of the side protective plate and to calculate the position of the parameters by show windows on left side of web page, helping designers intuitively understand the meaning of the parameter. By clicking on the mouse in the form of the drag, so that the virtual model can be observed from 360 degrees, three-dimensionally displays the exact position and size ratio of design parts. At the same time, in order to prevent losing the focus of attention from the drag, we design three visual angles view to observe the virtual model - the front, side, top view. Through the above method, the design of side protective plate can be better.

There are 5 functional keys on the page: calculate 1, calculate 2,store, check, and input.

calculate 1: For each guide rod force calculation, before input data for the calculation of data is to check data, if there is illegal or beyond the normal range of design data will be prompted to check 
data and re-input.

Calculate 2: the average force for each guide rod.

store: save the parameters in the input box to the database through the data interface for the next time usage.

Input: with different guide rod size, can be set to design the number of guide rods to 5 to 2 .

Check: after the total force of the guide rod is calculated, to check whether the force is appropriate.

The second section is the total force of the calculation, for the calculation of the side protective plate bearing force in the overall design. Calculating the total force is before to use that check function in the first section .

First of all, the calculation of total force need to read data calculated from hydraulic support optimization design integrated amongst the overall design platform. Data identifies through the interface, the interface converses the data to dictionary class data type and generates a temporary table, being automatically stored in cache, which is easily searched and speed up the processing speed of data. The virtual model of the calculation of the total force shows up in the display area after reading the data.

Virtual model in the display area of the total force web page is displayed from the whole frame level of hydraulic support, the picture is still support 360 degree design perspective, also marked the length and the position of the parameters on behalf of what meaning in actual support, is convenient observation of the design personnel.

(2) Telescopic beam design

The telescopic beam design displays in four sections: the telescopic beam, the total force calculation, simplified side view, force check.

The left part of the telescopic beam page is the virtual model display section. The right part is the function key and parameter input area.

The function keys are basically same as the side protective plate design, and from the front, the side, the top angles observation model, the receiving data from database.

The difference lies in the calculation of the force, the calculation of the trajectory, force check, the calculation of the three-dimensional trajectory, the calculation of swing force. These calculations buttons have the function of the preliminary checking data, will preliminarily check validity of data.

Total force calculation

The virtual display area of total force calculation page shows the position of the telescopic beams in the entire frame of support, as well as position of the input parameters. The "calculation" button is used to calculate the total force acting on a telescopic beam.

Simplified side view

Before using force calculation functions, it will test condition whether it fits the prerequisite, if it is not then will not be able to enter the design page. The design page of the above telescopic beam are relative, the step-by-step page design method makes the design work can be completed in stages, reducing the working intensity.

(3) Pushing mechanism design

The mechanism design displays in six sections: pushing mechanism 1,pushing mechanism 2, pushing mechanism 3,pushing mechanism 4, push rod parameter, joint parameter.

Pushing mechanism 1 to 4 calculates 4 kinds of pushing mechanism's situations . In addition to having the virtual model like other design modules, as well as pushing mechanism 1 has the virtual display of joint and box. A button named push mechanism can calculate the force of the push rod and the joint. After calculation, the shear force and bending moment diagram can be drawn. It can draw the normal force, the partial load, the lateral force, the stretching $1 / 3$, the stretching $2 / 3$, the stretching entire course, the shrinking $1 / 3$, the shrinking $2 / 3$, the shrinking entire course of the shear force and the bending moment diagram. Rendering these images do not need to enter any data, it makes use of the calculated data of push rod, data stored in static global temporary tables, for drawing a specific image to read data from temporary tables then put it into memory class, save in 
the cache, so that system can easily call.

After it has parameters of push rods and joint, whether joint being correct can be checked.

The push rod parameters is for the bear force calculation of push rod.

Joint parameters is for the bear force calculation of joints.

(4) Flipper with lemniscates design

Flipper with lemniscates design displays in four sections: flipper 1,flipper 2, the combination of flipper.

Flipper 1,the left side of page shows the three-dimensional diagram that indicates the position and length of these parameters. Motion simulation button at functional area can generate the animation of the flipper's movement, finish of animation demonstration followed by the output of the parameters of the simulation results, the designer can choose whether to output to excel file. Other buttons at the functional area, the bear calculation button is for the calculation of flipper's the bear force, the course calculation button is for the calculation of flipper's course. The check calculation shows that the bending moment, torsion moment, shear force, box section and provides the check function.

Flipper 2 is similar with flipper 1. Its addition is a multi-value calculation and optimal resistance calculation.

The combination of flipper is used to calculate two flippers. Input area to enter the first, the second parameters of flipper, meanwhile set they are pendulum bar mechanism or four bar mechanism, according to the different mechanism calculated their bear force. At the calculation, the gravity parameters of the two flippers bearing should be considered.

(6) Because the functions of other design modules and modules described above are in similar, it will be not in the repeat. Which linkage, machine selection, speed calculation, piping calculation are a single design unit, no subordinate design page.

\section{Integration effect}

After the actual test, 10 existing software combined in the overall design platform can co-work, data sharing, and meet the designers' need for simultaneous work. After the completion of the overall design, the virtual model of the components design platform gives design prototypes. Through the heterogeneous data interface, components design platform can well receive data from overall design platform, using received data for the design of parts. The basic data of parts are also well preserved in the server database, which prepares for future use or other designers to retrieve. Through the cooperation of the two platforms, designers can basically complete all kinds of calculation of the hydraulic support. With cooperation of the factory, after installation and repeatedly debugging, at present this design platform has been running working for more than a year's time, the feedback is good.

\section{Summary}

Using a number of technology: private cloud technology, web service, Mircosoft Silverlight, to achieve network and virtualization of hydraulic support design software, to solve problem like that 10 existing software can't be collaborative work, and shortage of parts design software and isolated data island. The hydraulic support design platform based on the existing software and B/S structure, it helps the designer to complete the design work from overall to part. However, the computer-aided design of the hydraulic support is engineering involved in many fields, knowledge, there are still many problems to be solved. For instances, in the design work of the hydraulic support problems between the realistic level of virtual imaging and system resource consumption, a wide variety of buttons in the software need to be reduced for more users-friendly. These problems still need to be further studied.

\section{References}


[1] Wei Qingtao,Ji Yonggang.Analysis and Study of impact of automation technology on development of advanced manufacturing technology[J].Electronic Test ,2014(3):95-96.

[2] Wu Lei. Research on Resource Virtualization in Manufacturing Grid[D]. Shandong: Shandong University,2008.

[3] Liu shijun,Wu Lei. Manufacturing Grid[M].Beijing: Publishing House of Electronics Industry,2007,31-32.

[4] Ji hai. Product Platform Design Services based on Cloud Computing[D].Beijing:China Academy of Machinery Science \& technology,2012.

[5] Zhong ruiming.Rich Cloud:Research on Implementation Mechanism for Mutual Backup Based Reliable Cloud Storage System across Heterogeneous Cloud Platforms[D].Beijing: Beijing University of Posts and Telecommunications, 2014.

[6] Information on http://www.chinasi.com/news/show-102.html.

[7]Information on

http://baike.baidu.com/link?url=a3C5OaJ8mwC33doyll4a-4Si618IMu-DKb6LoVJa4ZGzxnzgeizU e0AXALpAX-XvoCG9R2jwztK9pQZJtfN068zekqqJ5JJOZ0nNhQGKNkLTeV0Jalg0UChw_5yQ Dvs0bsbHseB1afP9D-a1Z4qF1_

[8] Yang jianzhou.Construction of Cloud Platform for Laboratory in University and Its Application Case[D].Shanghai:Donghua University,2014. 